Muñoz-Salgado, Nava-Rogel y Rangel Magdaleno (2013) / Revista de Empresa Familiar, 3(2), 29-41.

\title{
Capacidades innovadoras como estrategia de crecimiento en PYMES familiares.
}

\section{Innovative capacities as growth strategy in family SME.}

\author{
Fanny Janeth Muñoz-Salgado $^{\mathrm{a}} \cdot$ Rosa Maria Nava-Rogel $^{\mathrm{a}^{*}} \cdot$ Jorge Antonio Rangel Magdaleno $^{\mathrm{b}}$ \\ ${ }^{a}$ Universidad Autónoma del Estado de México (México) ${ }^{b}$ Universidad Autónoma de Aguascalientes (México)
}

\section{A T OS ARTÍCULO}

Historial:

Recibido 08-06-2013

Aceptado 30-10-2013

Palabras clave:

Empresa Familiar

Capacidades Innovadoras

Crecimiento Organizacional

PYMES Florícolas

Códigos JEL:

D13, O31, O43, O13

\section{A R T I C LE IN F O}

Article history:

Received 08-06-2013

Accepted 30-10-2013

Keywords:

Family Business

Innovative Capabilities

Organizational Growth

Floriculture SME

JEL codes:

D13, O31, O43, O13

\section{R E S U M E N}

Actualmente las capacidades innovadoras de las empresas familiares son un factor determinante para sobrevivir y obtener éxito, pues se convierten en una estrategia cuyo desarrollo y fortalecimiento depende de la gestión de la actividad innovadora realizada desde el interior de las organizaciones. Este estudio analiza el tipo de capacidades innovadoras presentes en mipymes familiares con el objeto de conocer el grado de contribución que brindan al crecimiento organizacional. Mediante un análisis exploratorio y descriptivo en pymes familiares productoras de flor en Villa Guerrero Estado de México, se encontró que las capacidades innovadoras incrementales impulsan en mayor grado el crecimiento organizacional de este tipo de empresas debido a su propia naturaleza.

* Autor de contacto.

Correos electrónicos: fanny.munozsalgado@gmail.com,rosanr02@hotmail.com,portizjorgerangel@um.es 


\section{Introducción}

La evolución de la literatura sobre "empresa familiar", como disciplina de carácter emergente, inicia en la década de los 60 aunque es 1980 el año en que diversos trabajos sobre empresa familiar abordan temas referentes a la economía y a la teoría organizacional (Benavides et al., 2011). Años más tarde, Wortman (1994) expuso la necesidad impostergable de generar investigación enfocada al desarrollo de teoría propia de la empresa familiar, hecho que fue el inicio de una nueva ola de estudios orientados a conocer las características y los fenómenos que se presentan en el interior de las organizaciones de esta naturaleza.

En este contexto, y bajo la perspectiva de la teoría de los recursos (Wernerfelt, 1984; Grant, 1991; Barney, 1991) como referente teórico en estudios de la empresa familiar (Sharma et al.1997; Cabrera, De Saá y García, 2001; Sirmon y Hitt, 2003; Moores, 2009; Debicki et al. 2009), se detecta el fenómeno del "familiness" o dotación de recursos específicos, que distingue a las empresas familiares, condicionada por la relación entre familia y empresa (Habershon y Williams, 1999; NuñezCacho y Grande, 2012). Sin embargo, la sola posesión del "familiness", como recurso valioso, raro y difícil de imitar, no es suficiente para obtener ventajas competitivas automáticamente (Sirmon y Hitt, 2003); más bien, la forma de generar verdaderas ventajas competitivas sostenibles está dada por las habilidades de las organizaciones para gestionar sus recursos en entornos cambiantes, mejor conocidas como capacidades dinámicas (Barney y Griffin, 1992; Teece, Pisano y Shuen, 1997; Peteraf, 1993; Penrose, 1959; Wernerfelt, 1984).

A partir de este planteamiento fundamentado en la teoría de los recursos y las capacidades dinámicas, surge el interés de diversos autores por estudiar tanto los recursos que provienen del interior de la empresa familiar como la dinámica de sus habilidades para gestionarlos (Cabrera Suarez et al., 2001; Chrisman et al., 2003; Kellermans y Eddleston, 2007; Cruz y Nordqvist, 2012), ya que ambos garantizan su sobrevivencia, crecimiento y diferenciación sostenible ( Habbershon y Williams, 1999; Habbershon et al., 2003; Chrisman et al., 2005). De igual manera, la revisión de la literatura del estudio de la empres familiar, destaca a la dirección estratégica y al cambio organizacional como tópicos preponderantes relacionados a la emprendeduría y a la innovación (Benavides et al., 2011); sin embargo, se identifica cierto vacío de la investigación en estudios de innovación en empresas familiares (Zahara y Shaman, 2004; Casillas y Acedo, 2007), aun cuando se ha demostrado la importancia estratégica de ésta a lo largo de la historia de la teoría organizacional con respecto al crecimiento (Shumpeter, 1934 y 1939), a la competitividad (Porter 1990) y al rendimiento (Capon et al., 1990; Hart 1992) de las organizaciones.

Por tal motivo, el presente estudio pretende contribuir al escaso cúmulo de investigaciones interesadas en el fenómeno de la innovación en empresas familiares (Craig y Moores, 2006; Craig y Dribell, 2006; Chen y Hsu, 2009; Waghner, 2010; Lorenzo y Nuñez-Cacho, 2012; López et al., 2012). El objetivo principal es analizar, mediante un estudio exploratorio, el tipo de capacidades innovadoras que surgen como estrategias de crecimiento al interior de empresas familiares floricultoras del Estado de México que les permiten la creación, posesión y mantenimiento de recursos generadores de valor y facilitadores del crecimiento.

El artículo presenta un primer apartado de revisión de la literatura para definir los conceptos de empresa familiar, capacidades innovadoras y crecimiento organizacional, con la finalidad de brindar un panorama del estado de la cuestión del tema en base a estudios anteriores. Posteriormente, el apartado de metodología explica la forma en que fueron aplicadas las entrevistas como técnica cualitativa para recabar información de primera mano gracias al contacto directo con dirigentes de mipymes familiares floriculturas del estado de México. El análisis de los datos mediante la codificación, permitió corroborar que las capacidades innovadoras incrementales son empleadas con mayor frecuencia y facilidad en mipymes familiares floricultoras por sus bajos 
costos y la facilidad de implementación, aunque el crecimiento organizacional se acelera bajo la presencia de las capacidades innovadoras radicales. El apartado de conclusión expone las limitaciones del estudio y propone nuevas líneas de investigación a considerar en el futuro.

\section{Revisión de la Literatura}

\subsection{Pyme familiar}

Pese a que cada país tiene diversos criterios para definir y clasificar a la PYME (Garza, 2000; Tunal, 2003 ; Zevallos, 2003; Saavedra y Hernández, 2008), término que comprende la micro, pequeña y mediana empresa, en México es considerada como aquella empresa que en su estructura no excede los doscientos cincuenta trabajadores y que percibe menos de dos cientos cincuenta millones de pesos mexicanos por producto de las ventas obtenidas en el transcurso de un año; dicha clasificación de empresas representa el noventa y nueve punto ocho por ciento del total de unidades económicas de México (Censos Económicos, 2009) y es considerada como la columna vertebral de la economía nacional por conformar la mayor parte del universo empresarial.

En una sociedad mexicana donde la institución principal es la familia transmisora de cultura, contenedora de roles sociales, forjadora de relaciones de poder y encargada de la formación de mando por vía de los integrantes que intervienen en su dirección y operación (Rangel y Moreno, 2012), es considerable concebir que cada negocio es un poco familiar y cada familia es un poco negocio (Aldrich y Cliff, 2003), de tal suerte que las estadísticas del último censo económico (2009) sustenta dicha idea bajo el entendido de que el noventa por ciento de las pymes mexicanas son consideradas empresas familiares.

Por definición la empresa familiar es aquella organización en la que al menos el $50 \%$ de su propiedad pertenece a una familia de la cuál algún miembro está a cargo de la dirección, gestión ó administración de la misma (Dyer, 2006). De acuerdo con Chrisman, Chua y
Sharma (2005), los negocios familiares son únicos, como consecuencia del involucramiento de los miembros de la familia en la propiedad, la gestión y la potencial transferencia generacional que se presenta cada 30 o 35 años. Dicha transferencia generacional es producto de un relevo en el mando que permite diferenciar la generación que se encuentra a cargo de la empresa; lamentablemente, sólo el 30\% de las empresas familiares sobrevive a la segunda generación y únicamente el $13 \%$ a la tercera (Ward, 1994).

Entre otras características que distinguen a las empresas familiares de aquellas que no lo son, se encuentran: la voluntad de estabilidad y perdurabilidad ante adversidades económicas relacionadas con la propiedad, el control y la gestión (Maseda, Iturralde y Arosa, 2009), la orientación al consumidor, el enfoque a la calidad y la activa participación dentro de las comunidades (Ibrahim, Angelidis y Parsa, 2008), ambiente de trabajo único (Hoffman et al., 2006) y especial manifestación de motivación y practicas eficientes (Levring y Moskowitz, 1993) que propician lealtad y confianza entre sus integrantes (Tagiuri y Davis, 1996).

Por otra parte, la empresa familiar como estructura orgánica conformada mayormente por individuos que comparten vínculos por consanguinidad, afinidad, adopción, matrimonio u otra relación estable de afectividad análoga (Real Academia Española, 2001), posee limitaciones organizacionales que no se hacen presentes en las empresas no familiares tales como: la práctica de una gestión complicada (Sirmon y Hitt, 2003), divergencia entre los objetivos debido a los valores de la familia y su influencia en el negocio (Cabrera, De Saá y García, 2001; Larraza et al. 2007), la presencia de nepotismo, paternalismo, temor a los cambios y finalmente la dependencia de financiamiento externo que limita su performance innovadora al interior (Lorenzo y Nuñez-Cacho 2012).

Investigaciones precedentes señalan que pese a la dotación de características y recursos específicos resultante de la relación entre familia y empresa (Habershon y Williams, 
1999; Nuñez-Cacho y Grande, 2012), mejor conocida como "familiness", sólo la habilidad para gestionar dichos recursos dentro de un entorno cambiante es la que le permite obtener ventajas competitivas sostenibles (Sirmon y Hitt, 2003; Barney y Griffin, 1992; Teece, Pisano y Shuen, 1997; Peteraf, 1993; Penrose, 1959; Wernerfelt, 1984); por lo que la unión de recursos específicos de la empresa familiar y las capacidades dinámicas desarrolladas al interior de la organización (Cabrera Suarez et al., 2001; Chrisman et al., 2003; Kellermans y Eddleston, 2007; Cruz y Nordqvist, 2012), garantiza la sobrevivencia, crecimiento y diferenciación sostenible ( Habbershon y Williams, 1999; Habbershon et al., 2003; Chrisman et al., 2005) de las empresas familiares. En este sentido, el presente estudio pretende identificar las "capacidades dinámicas innovadoras" características en pymes mexicanas con la finalidad de brindar evidencias empíricas que den soporte a la literatura relacionada al tema y contribuyan a consolidar una teoría propia de la empresa familiar.

\subsection{Capacidades innovadoras}

Con la finalidad de comprender lo que son las capacidades innovadoras se hace referencia primeramente a la definición del término innovar, que etimológicamente proviene del latín innovare, cuyo significado quiere decir cambiar o alterar las cosas introduciendo novedades (Real Academia Española, 2001). El término innovar representa un conjunto de actividades inscritas en un determinado tiempo $\mathrm{y}$ en un determinado lugar (Pavon y Goodman, 1981), que aplicadas a las organizaciones, conducen a introducir una idea en forma de nuevos o mejores productos, servicios, técnicas de gestión u organización con éxito.

Nelson y Winter (1982) consideran que en las empresas existe una base de rutinas para realizar tareas, actividades ó acciones que generan innovaciones mediante sus habilidades y capacidades para hacer, crear o modificar algo mejorándolo mediante la manipulación de los recursos que posee la empresa. Esta forma de gestión de los recursos se relaciona con la noción de capacidades dinámicas (Teece, Pisano y Shuen, 1997), misma que cuando está orientada a la gestión del cambio, al impulso emprendedor y a la creación de valor a lo largo del tiempo (Chirico y Nordqvist, 2010) puede considerarse como capacidad innovadora.

Las capacidades innovadoras proveen a la organización de herramientas de mejora, superación y crecimiento mediante cambios que mejoran los procesos o productos, incluyendo tanto los sistemas como los recursos empresariales, hecho que convierte a la capacidad innovadora en un potencial interno para generar nuevas ideas, identificar oportunidades comerciales y de mercado para apalancar los recursos y capacidades existentes en una organización (Hii \& Neely, 2000).

Abernathy y Clark (1985) identifican dos tipos de capacidades innovadoras centradas en un enfoque que atiende al grado de originalidad y novedad del proceso de innovación (Henderson y Clark, 1990; Damanpour y Gopalakrishnan, 2001; Tidd, 2001; Koberg, Detienne y Heppard, 2003; Subramanian y Youndt, 2005 y Stieglitz y Heine, 2007): incrementales y radicales, ambas determinadas por el tiempo de adopción que refleja la rapidez para generar o adoptar la innovación con relación a los competidores de la industria (Gopalakrishnan y Damanpour, 1997). La capacidad innovadora radical se entiende como un cambio rápido y la implementación de algo totalmente nuevo, mientras que la capacidad innovadora incremental se da bajo implementaciones dosificadas en los sistema, productos o procesos de la organización.

Como las capacidades innovadoras, implican la renovación del conocimiento o el uso diferente del viejo conocimiento para diseñar, crear y desarrollar nuevos productos, servicios, procesos o modelos de negocios con el propósito de crear valor para la organización (Santos-Rodrigues, 2010; Molina y Martínez, 2010; Green y Revilak, 2009) entonces se dice que las empresas que más rápido o temprano innovan, son las que tienen más propensión a conseguir verdaderas y durables ventajas competitivas (Birkinshaw y Mol, 2006; Porter, 
1980) y por lo tanto son generadoras de verdadera innovación organizacional.

Como se puede observar, la innovación organizacional no es necesariamente el resultado de departamentos estructurados de investigación y desarrollo, sino de los procesos informales o subcontratados (Sanchez, 2009) generados por nuevas formas de explotar, usar o combinar los recursos de la empresa (Goshal y Moran, 1996), la cual incluyen la mejora de la eficiencia y el control de las actividades organizacionales (Kong, 2010). Aun cuando las capacidades innovadoras no siempre son exitosas y el costo organizacional de los fracasos incluye: desperdicio de recursos en tecnología inapropiada, constante incertidumbre y baja moral empresarial debido a los infructuosos esfuerzos de las malas implementaciones (Johnson, 2010), tanto en recursos como en la habilidad para manejarlos, toda empresa debe desarrollar las capacidades innovadoras que le permitan generar una ventaja competitiva (Salavou, 2004) en el entorno que ella se sitúe, es decir, elegir estratégicamente lo que más le convenga para mejorar sus situación actual.

En el caso concreto de la empresa familiar se reconoce la importancia de la innovación como componente clave de su estrategia, en un sentido comparable a las empresas que operan en sectores de tecnologías avanzadas (Craig y Dibrell, 2006) el carácter innovador de la empresa familiar concede gran importancia a la capacidad innovadora. Sin embargo, la actitud frente al riesgo de algunas empresas familiares es de tipo conservadora manifestada por una aversión al riesgo (Fernández y Nieto, 2002) que determina el hecho de que unas empresas sean más innovadoras que otras. Los motivos principales que pueden bloquear el cambio en empresas familiares son: la inacción debido al temor de los directivos encargados de implantar el cambio de alterar el status quo existente dentro de la organización; las rutinas establecidas, la necesidad de abandonar prácticas establecidas para hacer cosas nuevas y desconocidas, que aporta una gran cantidad de inercia; los problemas de acción colectiva, esto es, la dificultad de movilizar en una dirección homogénea a toda una organización; y la carencia de las capacidades necesarias para afrontar el cambio con garantías de éxito (Rumelt, 1995; Lorenzo, 2003). Con el objeto de visualizar la forma en que las empresas familiares generan innovaciones, el presente artículo analiza las capacidades innovadoras que realizan como estrategia de crecimiento organizacional.

\subsection{Crecimiento organizacional}

Para referirse al término "crecimiento organizacional", tanto para la literatura económica como para la administrativa, se adoptan diferentes criterios de definición. En ocasiones se le considera como un objetivo deseado (Blázquez, Dorta y Verona, 2006), en otras como un proceso intangible que depende de una adecuada organización de elementos tangibles (capital físico, estructura interna y capital humano) (Cardona y Cano, 2005), algunas veces se entiende como un proceso social disciplinado e incluso en otras como un acto creativo que enlaza las diferentes partes móviles de una organización (Charan, 2004). Lo cierto es que pese a la diversidad de criterios, el denominador en común se resume a que el crecimiento organizacional es producto del manejo adecuado de recursos que poseen las organizaciones al interior, es decir, a la acción compleja de las modificaciones internas que una organización pueda tener y que desembocan en cambios de las características que identifican a una empresa.

Económica y financieramente, "crecer" es sinónimo del aumento en utilidades, y se considera al crecimiento organizacional como un índice del desempeño económico dinámico de la empresa que permite medir su capacidad así como ampliar la gama de oportunidades tanto comerciales como técnicas en los que la compañía puede incursionar y a las que antes no podía por no tener suficiente capacidad financiera (Sallenave, 1985). Si la empresa requiere de recursos financieros para crecer y estos recursos se originan al aumentar la demanda del producto o servicio, entonces la empresa recibirá mayores recursos que a su vez 
reinvertirá buscando aumentar la capacidad productiva para salir al mercado y así incrementar los recursos de capital que le permitirán crecer (Puerto, 2010); de esta manera se establecen las espirales de crecimiento como un sistema en el cual el crecimiento absoluto de un elemento provoca el crecimiento de otros elementos (Sallenave, 1985).

El crecimiento organizacional también hace referencia al desarrollo continuo que alcanza una empresa mediante una mayor aceptación de los productos y/o servicios que fortalecen su posicionamiento en los mercados de participación actual, ó bien, la expansión hacia nuevos mercados tanto geográficos como de clientes. Dicho índice de comportamiento dinámico de la empresa mide su aptitud para ensanchar sus posibilidades comerciales, financieras y técnicas en mercados con alto grado de dinamismo tecnológico a fin de conseguir un aumento constante de las ventas. De esta manera se asegura la supervivencia de la empresa al mantener paridad competitiva con sus rivales, desarrollar estrategias de inversión en innovación y desarrollo y emplear nuevas técnicas de productividad y marketing (Canals, 2000; Penrose, 1962; Puerto, 2010).

Como se puede observar, el crecimiento organizacional se caracteriza por tener presente la intervención de los diferentes sectores con los cuales sostiene relaciones una empresa y que pertenecen al ambiente externo de las organizaciones (proveedores, mercado financiero y de capitales, compradores, etc.), dicha condición afecta el crecimiento debido a la inexistencia de estímulos estatales, las características del financiamiento externo, el atraso tecnológico, la falta de adaptación a las condiciones cambiantes de los consumidores, la desviación de fondos de inversión y las variantes tendencias de mercado (Ortiz, 2005; Hernández y Mendoza, 2006).

Es por eso que los cambios que se pudieran ver reflejados en un aumento de las dimensiones del capital físico o del capital humano, en la participación del mercado, en el aumento de niveles de producción, ventas o utilidades gracias al adecuado manejo de los recursos internos para el proceso de crecimiento, considerado en ocasiones más o menos accidental, ocurren en un momento en que las condiciones favorecen la propia naturaleza del "organismo" (Penrose, 1962); en el caso de la pyme, se ha identificado que el proceso no solo requiere de cambios de naturaleza cuantitativa o tangible sino de cambios de naturaleza cualitativa o intangible que son de gran influencia para el desarrollo o surgimiento de los cambios positivos tan anhelados por los directivos de las organizaciones (Aguilera, 2010). Este estudio pretende entonces identificar la forma en que se generan los cambios en el proceso de crecimiento así como los resultados que se obtienen al término del proceso.

\section{Metodología}

Bajo la premisa de comprender y generar evidencia de casos múltiples conformado por micro y pequeñas empresas familiares, se presenta un estudio de carácter cualitativo que permitirá a la investigación comparar el estudio integral de cada una de las organizaciones ilustrando sus características y decisiones a partir de un determinado contexto (Yin, 2003).

El origen a la realización de un estudio exploratorio y descriptivo, surge de la inquietud por conocer un fenómeno contemporáneo en su propio contexto que permita mostrar la presencia e importancia de la administración estratégica en la innovación de diez pymes familiares productoras de flores ubicadas en Villa Guerrero Estado de México.

Las preguntas que el estudio pretende responder son: ¿Cuáles son las capacidades innovadoras distintivas y propias de la pyme familiar? $\mathrm{Si}$ bien las características de las empresas elegidas coinciden en cuanto a tamaño, ubicación geográfica, giro y estructura, la participación de ellas para el estudio fue voluntaria. La recopilación de datos se realizó mediante entrevistas con los dirigentes de las empresas florícolas, siendo dirigidos hacia la comprensión de las perspectivas que tienen al respecto de sus vidas, experiencias y labor empresarial, expresadas con sus propias palabras (Taylor y Bodgan, 1998). 
Los datos fueron analizados mediante la transcripción de las entrevistas en Word y bajo los criterios de González (2009): la conceptualización mediante la codificación de las capacidades innovadoras como guía para detectar cada idea aludida a un tópico en común, la categorización de las unidades de análisis correspondientes, la organización de las categorías por cada empresa en Excel y la estructuración por medio del cálculo de las frecuencias de las unidades de análisis por cada empresa de acuerdo a los códigos y categorías de las capacidades innovadoras detectadas en el estudio. Cada unidad de análisis estará representada por una oración ó párrafo que habla de un determinado tema y se identifican a partir de fragmentar las ideas que aluden a un tópico en común.

\section{Resultados}

Pese a la representatividad de las pymes familiares en la economía regional, las condiciones competitivas del mercado son muy difíciles de enfrentar, por lo que los lideres que están a cargo de su administración y dirección requieren contar con recursos tanto tangibles como intangibles que al momento de ser empleados en las actividades empresariales generen mejoras internas y externas reflejadas en la supervivencia y el crecimiento organizacional. En este sentido, y de acuerdo al análisis cualitativo de los datos obtenidos de diez entrevistas semi-estructuradas a dirigentes de pymes familiares productoras de flor en el Estado de México, se obtuvieron cinco categorías que identifican a las pymes como familiares: la propiedad familiar, liderazgo familiar, transferencia generacional, orientación y clima organizacional. Por la parte de las capacidades innovadoras, se identificaron sólo dos categorías que se hacen presentes mediante las capacidades innovadoras radicales como las incrementales, siendo éstas últimas las que son más implementadas por parte de los dirigentes debido a la facilidad para implementarlas. Finalmente del crecimiento organizacional se obtuvieron dos categorías que indican el reflejo del crecimiento en forma interna y externa de la organización, ambas visibles y provenientes de la buena o mala administración de los recursos tangibles e intangibles por parte de los dirigentes de la pymes familiares.

\subsection{Familiness}

De acuerdo a las entrevistas realizadas, de diez pymes familiares del sector floricultor en el Estado de México la propiedad de cuatro de ellas pertenecen en un $80 \%$ a la familia ascendente del dirigente a cargo, cuatro pertenecen en un $60 \%$ a la familia descendiente del dirigente con él a la cabeza y dos de ellas pertenecen en un $100 \%$ solamente al dirigente. El grado de estudios de los dirigentes resultó interesante debido a que, únicamente uno de ellos cuenta con estudios superiores con el título de ingeniero agrónomo, dos terminaron estudios de nivel medio superior, tres cuentan con certificado de secundaria y cuatro de ellos solo concluyeron la primaria, lo que indica que el grado de estudios de los dirigentes de este tipo de organizaciones no es un factor que limite su gestión.

Referente a la experiencia y conocimiento del negocio cuatro dirigentes llevan 20 años a cargo del negocio siendo la segunda generación familiar que después de 30 años, con su antecesor al poder, adoptaron la responsabilidad de la dirección de la empresa.

Uno de los líderes lleva 15 años al frente de su empresa aunque la empresa le fue heredad por su padre que fundó la empresa y que la mantuvo en operaciones por cuarenta años. Dos dirigentes llevan a cargo de sus negocios ya 10 años aunque antes de obtener su cargo tuvieron que pasar 25 años precedidos por otro miembro de la familia al frente de la empresa. Dos dirigentes más, llevan cinco años a cargo de su negocio siendo ellos mismos los fundadores de las empresas. Por último, uno de los dirigentes lleva un año a cargo de la empresa pero ya conocía el negocio puesto que ha trabajada en él toda su vida bajo la dirección primeramente de su abuelo durante quince años y de su padre durante veinte años hasta el momento en que le tocó tomar el poder.

Todos los dirigentes sin excepción dan testimonio de hacer uso de estrategias reactivas, 
es decir que realizan su gestión y administración orientando los esfuerzos de la empresa hacia las demandas del cliente consumidor. Sin embargo, dos empresas se enfocan a la especialización de su producto y la mejora de la calidad del mismo, dos empresas a la diversificación de productos $\mathrm{y}$ búsqueda de nuevos mercados, cuatro se enfocan únicamente a mantener satisfechos a sus clientes y dos unen esfuerzos por incrementar sus relaciones con otros organismos para promover sus productos. El clima organizacional que se vive en las diez empresas coincide en que está integrado por el sentimiento de confianza, lealtad y gran motivación gracias al interés por perdurar en el negocio de una forma estable y con expectativas de ser mejor día a día.

\subsection{Capacidades innovadoras incrementales $y$ crecimiento organizacional interno.}

Las diez empresas objeto de estudio para esta investigación aceptaron que de forma permanente se establecen rutinas o actividades para mejorar "poco a poco" los procesos de producción y los procedimientos administrativos al interior de las organizaciones. Dichos cambios y actividades implementadas en forma dosificada se traducen en las capacidades innovadoras incrementales que son empleadas de diversas maneras dependiendo las necesidades que la organización requiera satisfacer. Para efectos del estudio a cada dirigente de las empresas se les solicitó mencionar aquellas acciones o innovaciones que hayan realizado de manera paulatina y los beneficios que se obtuvieron con ellas con lo que se capturó la siguiente información.

Se obtiene mayor flexibilidad de los procesos de producción al momento de: mantener en orden las áreas de trabajo que van desde los invernaderos, oficinas, áreas de empaque, las zonas de carga y descarga, etc. Registrar los cambios en los procesos de suministro de insumos, refrigeración y procesos productivos en general. Los dirigentes establecen que los costos pueden reducirse si poco a poco se va cumpliendo con una planeación de la producción y la cuantificación de los insumos que se requieren para ella. Mantener comunicación vía telefónica con los proveedores y clientes para hacer de su conocimiento los cambios o requerimientos específicos de la empresa y por consiguiente mejorar los periodos de respuesta de ambos también es una capacidad innovadora que hace más flexible su empresa.

Los dirigentes afirman que al cambiar poco a poco la forma de comunicarse al interior de la organización disminuye la rotación de personal e incrementa la unión y lealtad de los colaboradores en ella, por lo que se han implementado pequeñas reuniones de trabajo en diversas horas del día para lograr el objetivo. Incluso mediante juntas de integración y comunicación se ha facilitado motivar al personal y generar en éste eficiencia y eficacia en sus labores. De tal suerte que se observa entonces que las actividades novedosas que se implementan en forma paulatina o dosificada genera mejoras e incluso crecimiento al interior de la empresa.

\subsection{Capacidades innovadoras radicales y crecimiento organizacional externo.}

Por medio del contacto directo con los dirigentes de las diez pymes familiares florícolas de Villa Guerrero estado de México, se encontró que no es frecuente que se generen cambios rápidos y totalmente nuevos en los procesos o procedimientos de las pymes familiares. Es decir, que la presencia de las capacidades innovadoras radicales únicamente se hace presente si el dirigente decide ampliar la gama de productos por el hecho de tener que allegarse de material genético totalmente nuevo, cuyo cuidado y proceso de producción será totalmente diferente al que se maneja generalmente en la empresa. Incluso, si se quisiera mejorar la calidad del producto que la empresa ya maneja entonces es necesario aplica técnicas de cuidado, fumigación y riego más sofisticadas que en ocasiones requieren innovaciones tecnológicas que por lo general no están al alcance de las posibilidades financieras de las pymes. 
Con la implementación de nuevos recursos de promoción de bajo costo, como las redes sociales y participación en eventos públicos, se incursiona en nuevos mercados que la mayor parte de las veces resultan más exigentes con los productores pero que provocan incrementos en la cartera de clientes, por consecuencia las ventas también tienden a incrementar y como resultado se obtienen mejores ganancias financieras que en el largo plazo son generadoras de implementaciones de tecnologías más sofisticadas.

En el caso de aquellos productores que en algún momento toman la decisión de invertir en maquinaria y equipo más sofisticado o de mejorar la calidad tecnológica de sus instalaciones, se da por hecho un visible crecimiento reflejado en las dimensiones de la infraestructura de su empresa pero afirman que en ocasiones resulta contraproducente, puesto que no obstante con haber perdido parte importante de su capital financiero resulta que la capacidad instalada a la que pueden llegar resulta desaprovechada en el largo plazo.

En atención a lo anterior se visualiza que el cambio radical que puede darse en las pymes familiares generan crecimiento visible al exterior de la empresa aunque los líderes afirman que por las características de la empresa es difícil adquirir o implementar cambios abruptos en ella y que si se analiza con minuciosidad, se puede caer un gastos que a la larga resulten infructuosos debido a las condiciones de comportamiento del mercado.

\section{Conclusión}

Cuando se entiende que las pymes familiares son organizaciones con especiales características a causa de los recursos que la conforman, el crecimiento tanto al interior como al exterior de las organizaciones se genera mediante la administración de dichos recursos y las habilidades que sus dirigentes tienen para hacer, crear o modificar algo de la organización. Esto es, mediante las capacidades innovadoras como estrategias de crecimiento, los líderes de pymes familiares de economías en vías de desarrollo generan diferentes estrategias alternativas para que sus empresas sobrevivan y crezcan.

Las capacidades dinámicas innovadoras más empleadas por parte de pymes familiares cuyos líderes no rebasan la tercera generación al mando, con estudios de nivel básico en su mayoría pero con gran experiencia y conocimiento del negocio que se encontraron fueron las incrementales. Dichas capacidades incrementales, entendidas como el conjunto de habilidades y actividades para hacer, crear o modificar algo mejorándolo mediante la manipulación paulatina de los recursos que posee la empresa, generan cambios principalmente al interior de las organizaciones, creando mayor flexibilidad en la producción, la reducción del tiempo de repuesta en clientes y proveedores, reducciones en costos e incrementos estructurales así como mejor comunicación en la empresa que brinda un ambiente de lealtad con colaboradores motivados para hacer sus labores en forma eficaz y eficiente.

Por otra parte también se observan las capacidades innovadoras radicales que implican cambios abruptos y definitivos en diversas áreas de la empresa, mismas que generan crecimiento visible al exterior de las pymes como tecnificación y el aumento de las dimensiones en infraestructura, una gama más amplia de productos con mayor calidad, un aumento de la participación en el mercado en el que se compito o bien incursionar en nuevos mercados mediante mejoras en las técnicas de promoción que generan incrementar la cartera de clientes y por ende las ventas y utilidades de la pyme familiar.

A partir de lo anterior, también se concluye que las capacidades innovadoras incrementales son las más practicadas por las pymes familiares, debido a la facilidad y bajos costos de implementación. Por el contrario, las capacidades innovadoras radicales son usadas moderadamente siempre que se trate a nivel de producto o promoción y casi de manera nula si se trata de su aplicación en la infraestructura puesto que los altos costos de inversión en ella se convierten, en el largo plazo, en capacidad instalada desperdiciada. 
El estudio presenta limitaciones debido a la naturaleza de la investigación, ya que como abordaje cualitativo carece por el momento de abordaje cuantitativo, mismo que se sugiere considerar para investigaciones futuras. Otra área de oportunidad identificada en la investigación es el análisis del contexto que enfrentan la pymes en economías en vías de desarrollo y la identificación de aquellos factores que inciden en el comportamiento de dichas organizaciones.

\section{Bibliografía}

Abernathy, W. J., y Clark, K. B. (1985). Innovation: mapping the winds of creative destruction. Research Policy, 14, 3-22.

Aguilera, A. (2010). Direccionamiento estratégico y redireccionamiento empresarial: algunas reflexiones en torno a su relación. Pensamiento \& Gestión, núm. 28, enero-junio, 2010, pp. 85-106. Universidad del Norte Colombia.

Aldrich, H.E. y Cliff, J.E. (2003). The pervasive effects of family on entrepreneurship: Toward a family embeddedness perspective. Journal of Business Venturing, 18, 573-596.

Barney, J. B. y Griffin, R. (1992). The management of organizations: Strategy, structure, behavior. Boston: Houghton Mifflin.

Barney, J.B. (1991). Firm resource and sustained competitive advantage. Journal of Management, 17, 395-410

Benavides, C.A.; Guzmán, V.F. y Quintana, C. (2011). Evolución de la literatura sobre empresa familiar como disciplina científica. Cuadernos de economía y dirección de la empresa, 14:78-90.

Birkinshaw, J. y Mol, M. (2006): How Management Innovation Happens, M.I.T. Sloan Management Review, vol. 47, n. 4, pp. 81-87.

Blázquez, F.; Dorta J. A. y Verona, M. C. (2006). Concepto, perspectivas y medida del Crecimiento empresarial. Cuadernos de Administración PUJ, 19 (31), 172, 167, 174-180, 176, 177,180-187.

Cabrera-Suárez, K., De Saá-Pérez, P., y GarciaAlmeida, D. (2001). The succession process from a resource- and knowledge-based view of the family firm. Family Business Review, 14, 37-46.
Canals, J. (2000). Crecimiento empresarial: personas y tecnología en la nueva economía. Revista Empresa y Humanismo, 2 (2), 337-370.

Cardona, M. y Cano C. A. (2005). Territorio, ciclo de vida y estructura empresarial: un puente en la industrialización regional. Medellín: Fondo Editorial Universidad EAFIT.

Censos Económicos (2009). Micro, pequeña, mediana y gran empresa: estratificación de los establecimientos: Censos Económicos 2009 / Instituto Nacional de Estadística y Geografía. México: INEGI.

Charan, R. (2004). El crecimiento rentable, un asunto de todos: 10 herramientas prácticas para el crecimiento empresarial. España: Empresa Activa.

Chirico, F. y Nordqvist, M. (2010). Dynamic capabilities and trans-generational value creation in family firms: The role of organizational culture. International Small Business Journal, 28(5), 487504.

Chrisman, J. J., Chua, J. H., y Steier, L. P. (2003). An introduction to theories of family business. Journal of Business Venturin. 18, 441448.

Chrisman, J.J., Chua, J.H. y Sharma, P. (2005). Trends and directions in the development of a strategic management theory of the family firm. Entrepreneurship Theory and Practice, 29, 555-575.

Craig, J. y Dibrell, C. (2006). The natural environment, innovation, and firm performance: A comparative study. Family Business Review, 19(4), 275-288.

Cruz, C., y Nordqvist, M. (2012). Entrepreneurial orientation in family businesses: A generational perspective. Small Business Economics, 38, 33-49.

Damanpour, F. y Gopalakrishnan, S. (2001): The dynamics of the adoption of product and process innovations in organizations, Journal of Management Studies, vol. 38, n. 1, pp. 45-65.

Debicki, B.J., Matherne, C.F., Kellermanns, F.W. y Chrisman, J.J. (2009). Family Business Research in the New Millennium: An Overview of the Who, the Where, the What, and the Why. Family Business Review, 22 (2), 151-166 Family Business Review, $22,167-180$.

Dyer, W. G. (2006). Examining the "Family Effect" on Firm Performance. Family BusinessReview, 19 (4), 253-273. 
Fernández, Z. y Nieto, M.J. (2002). La estrategia de internacionalización de la pequeña empresa familiar. Series de Economía de Empresa, Documento de Trabajo 02-18(11), 1-26.

Garza, C. R. (2000). Creacion de PYMES: Objetivo Emprendedor. Ingenierias, Vol. 3, No. 9, pp. 54-58.

Ghoshal, S. y Moran, P. (1996). Bad for practice: A critique of transaction cost theory. Academy of Management Best Paper Proceedings, J 7-21.

González, L. (2009). La sistematización y el análisis de los datos cualitativos. Tras las vetas de la investigación cualitativa, México: ITESO, pp. 158-173.

Gopalakrishnan, S. y Damanpour, F. (1997). A Review of innovation research in economics, sociology and technology management. Omega, vol. 25 , n. I, pp. 15-28.

Grant, R.M. (1991). The Resource-Based Theory of Competitive Advantage: Implications for Strategy Formulation. California Management Review, 33 (3), 114-135.

Green A. y Revilak A. (2009). Measuring Innovation from the Source to the Value. Proceedings of the European Conference on Intellectual Capital. Business Source Complete, Ipswich, MA.

Habbershon, T., y Williams, M. (1999). A resource-based framework for assessing the strategic advantages of famly firms. Family Business Review, $12,1-25$.

Habbershon, T.G., Williams, M.L. y McMillan,I.C. (2003). A unified systems perspective of family firm performance. Journal of Business Venturing, 18, 451-465.

Henderson, R. M. y Clark, K. B. (1990). Architectural Innovation: The reconfiguration of existing product tecnologies and the failure of established firms. Administrative Science Quarterly, vol. 35, n. 1, pp. 9-30.

Hernández, M. y Mendoza, J. (2006). Cultura organizacional, el caso de las empresas hidalguenses. Revista Internacional La Nueva Gestión Organizacional, 2(3), 137-162.

Hii, J. y Neely, N. (2000). Innovative capacity of firms: on why some firms are more innovative than others, Paper presentado en 7th International Annual EurOMA Conference 2000, Ghent.
Hoffman, J., Hoelscher, M. y Ritch, S. (2006). Achieving Sustained Competitive Advantage: A Family Capital Theory. Family Business Review, 19(2), 135-145.

Ibrahim, N.A., Angelidis, J.P. y Parsa, F. (2008). Strategic management of family businesses: Current findings and directions for future research. International Journal of Management, 25(1), 95110.

Johnson, J. D. (2010). Success in innovation implementation. Journal of Communication Management, Vol. 5, No. 4, 2001, pp. 341-359.

Kellermanns, F. W. y Eddleston, K. A. (2007). A family perspective on when conflict benefits family firms performance. Journal of Business Research, 60(10), 1048 - 1057.

Koberg, C. S., Detienne, D. R. y Heppard, K. A. (2003). An Empirical Test of Environmental, Organizational, and Process Factors Affecting Incremental and Radical Innovation. Journal of High Technology Management Research, $\mathrm{n}^{\circ}$ 14, págs. 2145.

Kong, E. (2010). Innovation processes in social enterprises: an IC perspective. Journal of Intellectual Capital. Bradford: Vol. 11, Iss. 2, p. 158-178

Larraza, M., Wiseman, R. M., Gómez-Mejía, L.R. y Welbourne, T. M. (2007). Disentangling compensation and employment risks using the behavioral agency model. Strategic Management Journal, 28(10), 1001-1019.

Levering, R. y Moskowitz, M. (1993). The 100 Best Companies to Work For in America. (V ed.). New York: V Plume

Lorenzo Gómez, J.D. (2003). El cambio estratégico en las organizaciones. Servicio de Publicaciones de la Universidad de Cádiz.

Lorenzo, J.D. y Núñez-Cacho, P. (2012). Inercia e innovación en la empresa familiar: una primera aproximación. Revista de Empresa Familiar, 2(2), 23-40.

Maseda, A., Iturralde, T. y Arosa de la Torre, B. (2009). Situación de la empresa familiar en el territorio histórico de Bizkaia. Ponencia presentada al XXIII Congreso Anual y XIX Hispano Francés de la Academia Europea de Dirección y Economía de la Empresa (AEDEM), Sevilla, España: ESIC.

Molina, F. X. y Martínez, M.T. (2010). Social Networks: Effects of Social Capital on Firm 
Innovation. Journal of Small Business Management; abril; 48, 2; ABI/ INFORM Global, pg. 258-279.

Moores, K. (2009). Paradigms and Theory Building in the Domain of Business Families.

Nelson, R.R. y Winter, S.G. (1982). $A n$ Evolutionary Theory of Economic Change, The Belknap Press of Harvard University Press, Cambridge, Massachusetts.

Nuñez-Cacho, P. y Grande. F. A. (2012). Family businesses: How to measure their performance. African Journal of Business Management, 6(12), 4612-4621.

Ortiz, G. A. (2005). Gerencia financiera $y$ diagnóstico estratégico. (2a ed.). Bogotá: McGrawHill.

Pavón, J. y Goodman, R. A. (1981) Proyecto MODELTEC. La planificación del desarrollo tecnológico, CDTI-CSIC, Madrid.

Penrose, E. (1959). The Theory of the Growth of the Firm, Wiley, Nueva York.

Penrose, E. T. (1962). Teoría del crecimiento de la empresa. Madrid: Aguilar.

Peteraf, M. (1993). The Cornerstones of Competitive Advantage: A Resource- Based View, Strategic Management Journal, vol.14, pp. 179-191.

Porter, M. E. (1980). Competitive Strategies: Techniques for Analyzing Industries and Competition, The Free Press, New York.

Puerto, D. (2010). La globalización y el crecimiento empresarial a través de estrategias de internacionalización. Pensamiento \& Gestión, núm. 28, enero-junio, 2010, pp. 171-195 Universidad del Norte

Rangel Peña, S. y Moreno Gomez, S. (2012). Protocolo de investigación: las PYMES y su eficacia en México. Observatorio de la Economía Latinoamericana, $\mathrm{N}^{\circ} 175,2012$. Texto completo en http://www.eumed.net/cursecon/ecolat/mx/

Real Academia Española. (2001). Diccionario de la lengua española (22.a ed.). Consultado en http://www.rae.es/rae.html

Rumelt, R.P. (1995). Inertia and transformation. En MONTGOMERY, C.A. (1995). Resource-Based and Evolutionary Theories of the Firm: Towards a Synthesis (pp 101-132). Kluwer Academic Publishers

Saavedra, M. L. y Hernández, Y. (2008). Caracterización e importancia de las MIPYMES en
Latinoamérica: Un estudio comparativo. Actualidad Contable Faces. Venezuela: Universidad de Los Andes, año 11, no. 17, julio-diciembre, pp. 122-134. [En línea] Disponible en: $<$ http://www.saber.ula.ve/bitstream/123456789/2662 8/1/articulo10.pdf $>$, consultada: mayo de 2013.

Salavou, H. (2004). The concept of innovativeness: should we need to focus?. European Journal of Innovation Management, Vol. 7, N. 1, pp. 33-44.

Sallenave, J. (1985). Gerencia y planeación estratégica. Ed. Norma México

Sánchez, G. (2009). Las micro y pequeñas empresas mexicanas ante la crisis del paradigma económico de 2009, Edición electrónica gratuita. Texto completo en www.eumed.net/libros/2009a/524/

Santos Rodrigues, H.P. (2010). The Influence Of Human Capital On The Innovativeness Of Firms. The International Business \& Economics Research Journal; Setiembre; 9, 9; ABI/ INFORM Global, pg. 53-64.

Sharma, P. Chrisman, J.J. y Chua, J.H. (1997).Strategic management of family business: Past research and future challenges. Family Business Review, 10 (1), 1-35.

Sirmon, D. G., y Hitt, M. A. (2003). Managing resources: Linking unique resources, management, and wealth creation in family firms. Entrepreneurship Theory and Practice, 27, 339-358.

Stieglitz, N. y Heine, K. (2007). Innovations and the Role of Complementarities in a Strategic Theory ofthe Firm, Strategic Management Journal, vol. 28, págs. 1-15.

Subramaniam, M. y Youndt, M. (2005). The Influence of Intellectual Capital on the Types of Innovative Capabilities. Academy of Management Journal, 48(3), 450-463.

Tagiuri, R. y Davis, J. (1996). Bivalent Attributes of Family Business. Family Business Review, 9(2),199-208.

Taylor, S. J. y R. Bodgan. (1998). Introducción a los métodos cualitativos de investigación. La búsqueda de significados. Barcelona, Buenos Aires:Paidós.

Teece, D.J., Pisano, G. y Shuen, A. (1997). Dynamic Capabilities and Strategic Management Strategic Management Journal, 18 (7), 509-533. 
Tidd, J. (2001). Innovation management in context: environment, organization and performance. International Journal of Management Review, vol. 3, n. 3, pp. 169-183.

Tunal, G. (2003). El problema de clasificación de las microempresas. En: Actualidad Contable FACES,Ano 6, No. 7, pp. 78-91.

Ward, J.L. (1994). Cómo desarrollar la empresa familiar. Buenos Aires: El Ateneo.

Wernerfelt, B. (1984). A Resource-based View of the Firm. Strategic Management Journal, 5, 171180.

Wortman, M. S. (1994). Theoretical foundations for family-owned businesses: A conceptual and research based paradigm. Family Business Review, 7(1), 3-27.

Yin, K. R. (2003). Case study research. Design and methods, USA: Research Methods Series.

Zahra, S. A., y Sharma, P. (2004). Family business Research: a strategic reflection. Family Business Review, 17(4), 331-346.

Zevallos E. (2003). Micro, Pequeñas y Medianas Empresas en America Latina. Revista CEPAL, No. 73 , abril. 